\title{
Balancing business with clinical excellence
}

Streamline your workflow and enhance patient care with Carestream Dental's innovative Sensei Cloud - the latest true cloud-based practice management platform.

Acting as a trusted digital mentor, Sensei Cloud is optimised to analyse your practice data, report on key performance indicators, and provide actionable steps that you can take to boost revenue.

It combines an intuitive design with powerful imaging, practice management and clinical workflow capabilities in one cloud-based platform, which can be accessed anywhere, any time for ultimate flexibility.

With real-time dashboards, revenue cycle management, built-in scalability and a variety of other invaluable tools, Sensei Cloud enables you to balance business with clinical excellence.

For more information contact Carestream Dental on 08001699692 or visit www.carestreamdental.co.uk.

\section{(n) Sensei}

\section{Cloud}

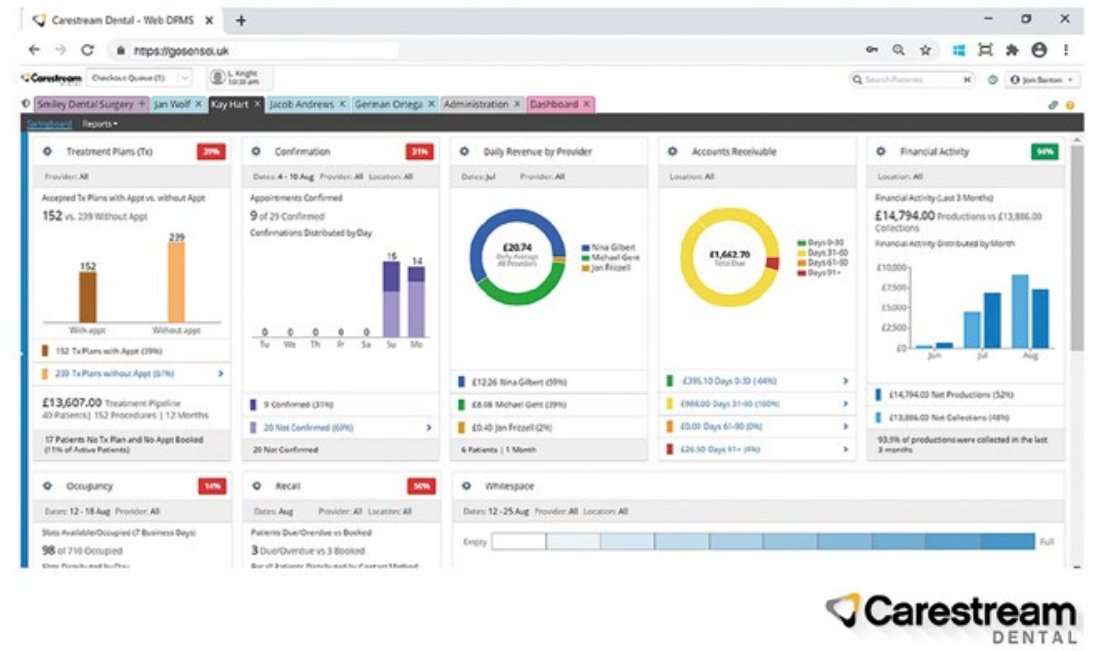

\section{Safe surfaces made easy}

In a dental practice, bacteria and viruses on surfaces are a real threat.

Protect your patients and staff alike with AzoMax Universal wipes from Eschmann.

A simple, stress-free way to achieve an excellent standard of surface disinfection, the wipes eliminate $99.9999 \%$ of pathogens using an alcohol-free formula that can be used on even delicate equipment.

Furthermore, as AzoMax Universal wipes are versatile enough to clean and disinfect surfaces and non-invasive medical devices, they can help you replace a wide range of cleaning products, saving you money without compromising the safety of your patients and staff.

For more information on the highly effective and affordable range of decontamination equipment and products from Eschmann, please visit www.eschmann.co.uk or call 01903 875787.

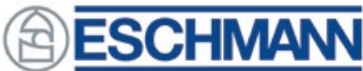

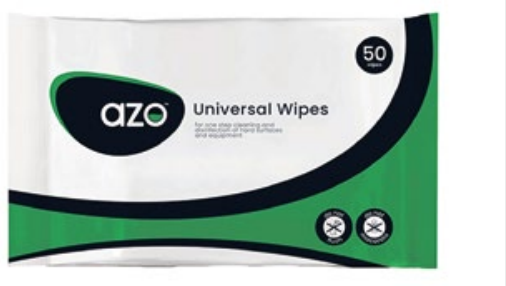

\section{An enhanced workflow}

Align Technology, Inc. (NASDAQ: ALGN) has launched an enhanced laboratory workflow, designed to significantly improve the collaboration between dental practices and laboratories, and can lead to increased efficiency, better accuracy, enhanced communication and a faster turnaround for both parties.

At the heart of the new workflow sits the cloud-based MyiTero.com platform, which enables the laboratory to receive scans within minutes, allowing them to review and communicate with the dentist in real time, for example while the patient is still in the chair. It provides the laboratory with increased flexibility to manage the restorative workflow, ultimately enhancing patient communication and outcomes, and reducing the need for multiple revisits.

The new workflow allows technicians to work on immediate scan enhancements and make timely modifications to their milled models, saving at least a day, if not more, on cases. Scans can be received and reviewed within minutes, rather than the previous timeline of 8-48 hours.

Coming soon, the productivity of the MyiTero platform will increase for laboratory users of exocad design software. Users of both the MyiTero platform and exocad software will be able to pair their accounts to allow the automatic import of iTero scans and prescription data into exocad DentalDB, eliminating the step of exporting cases from MyiTero.com. Key MyiTero features such as 'return case to doctor' will be available from exocad DentalDB while iTero services and 3D case preview functionality can launched from the same case management screen.

The new workflow is now available across UK and Ireland, Germany, Austria, Switzerland, Italy and across other countries in Europe, Middle East \& Africa.

For more information, please visit www. iTero.com or contact your local iTero representative. 